\title{
探讨人工智能在计算机网络技术中的应用
}

\author{
孙本楠 \\ 北京东方计量测试研究所，北京 100086
}

[摘要] 随着我国经济的快速发展,科学技术领域也获得诸多的成果,其中网络科学技术领域的发展更加瞩目,该技术在我国社会的 多个领域得到了广泛的应用。随着计算机网络技术应用的不断普及,计算机网络信息安全和系统管理等问题成为了社会各界关注 的焦点问题。而且,随着计算机网络技术的快速发展,计算机网络信息安全管理以及系统管理中应用的各种问题求解功能,已经不 能满足当下时代的发展需求,而人工智能在计算机网络技术中的应用,显然为此类问题提供了一个创新的解决途径。而且,通过人 工智能的介入, 能够有效提高网络资源利用率, 同时,还可以对各种复杂的网络资源进行高效的梳理和有效的甄别, 从而使网络技术 的应用效果得到进一步提高。

[关键词]计算机网络技术, 人工智能,作用

\section{Discussion on the Application of Artificial Intelligence in Computer Network Technology}

\author{
SUN Bennan
}

Beijing Dongfang Metrology Testing Research Institute, Beijing,China, 100086

\begin{abstract}
With the rapid development of China's economy, many achievements have been made in the field of science and technology, in which the development of network science and technology is more and more attention, and the technology has been widely used in many fields of our country. With the popularization of the application of computer network technology, the security of computer network information and system management have become the focus of attention from all walks of life.Moreover, with the rapid development of computer network technology, all kinds of problem solving functions applied in computer network information security management and system management can no longer meet the development needs of the present era. The application of artificial intelligence in computer network technology obviously provides an innovative way to solve this kind of problem.Moreover, through the intervention of artificial intelligence, the utilization rate of network resources can be improved effectively, at the same time, various complex network resources can be effectively combed and effectively screened, so that the application effect of network technology can be further improved.
\end{abstract}

Keywords: Computer network technology;Artificial intelligence;Function

引言

人工智能不但给人们的日常生活带来了非常大的便利，且在计算机网络中得到了非常广泛的应用，同时，人工 智能的发展也离不开计算机网络的支持，两者具有着相辅相成的关系，能够实现共同发展、共同进步。

\section{1 人工智能概述}

人工智能也可以称作为机器智能，就是采用相关科技手段，向机器输入类似于人类的一些行为、意识以及思考 指令，从而使机器变得如人一般智慧，然后再让其代替人类去完成一些人类无法完成或危险性较高的事情。人工智 能的特点主要有以下几点：能对信息进行高效的处理、推理以及学习能力强等。随着人工智能的快速发展，人们也 逐渐开始了将人工智能应用在计算机网络技术中的实践，这一实践其实是具有一定可行性的，主要体现在以下几个 方面：

第一，人工智能可以对不确定信息进行准确的处理，这是人工所不能及的，同时，它还可以实现对系统资源的 有效掌握与追踪，且信息的处理效率非常高，能够更加及时的向用户提供准确信息。

第二, 人工智能可以对复杂的信息数据进行快速的整理与分类, 并以此为基础 , 实现信息资源的共享与高效传 输，所以说，在计算机网络技术中合理应用人工智能，能够使信息处理效率得到有效提高。

第三, 人工智能本身具有着非常高的学习以及推理能力, 将其应用在计算机网络技术中, 可以有效促进计算机 
网络管理质量及管理效率的提升。

第四, 人工智能具有较高的记忆能力, 能够快速完成信息库的建立, 这对于计算机网络管理工作来说有着很大 的促进作用。此外, 人工智能还可以实现对非线性问题的有效处理。可见, 将人工智能应用在计算机网络技术中是 具有一定的可行性的。

\section{2 计算机网络技术存在的问题}

互联网技术的迅速普及和各类智能操作设备的发明, 使得计算机网络技术的触角遍布人类社会的每个角落, 人 们已经适应了网络技术无处不在的便捷和高效。但随之而来的是, 随着人们越来越多的将生活场景搬到网络上, 个 人信息的安全就成了至关重要的问题, 如何确保个人信息的安全, 如何确保自身获得的信息是正确的, 这很难通过 人工手段去甄别和防范, 这时候就需要人工智能技术的介入。通过人工智能技术的介入, 可以迅速的甄别各类虚假 信息，并拦截绝大多数的恶意攻击和未经授权的访问。随着人工智能技术的应用逐渐推广，人工智能自身也积累了 海量的数据信息, 从而愈发灵敏和智能化, 对网络安全的防护能力也不断强化, 因此, 人工智能在互联网计算机技 术中的应用也越来越广泛。

\section{3 计算机网络技术中人工智能的应用}

\section{1 计算机网络安全方面人工智能的应用}

（1）将人工智能应用在计算机网络技术中后，能够使计算机的整体功能得到有效提升，能够更好的满足人们对 计算机信息的需求，智能化的网络技术服务能够更加便捷和高效，主要是体现在人机交互界面的智能化、后台技术 开发的智能化以及系统支撑环境的智能化调节等方面，从而全面提升网络技术的服务水平，尤其是在网络信息安全 防护领域，智能化的应用能够使信息安全得到更加有效的保障。将人工智能应用在计算机网络技术中，可以利用人 工智能系统, 来对大量的信息数据进行高效处理, 并从中找出真实性、可靠性以及价值性较高的信息, 这样能够有 效提高信息的安全性。同时，人工智能系统还可以对计算机网络信息进行高效的检测，能够及时发现不安全信息并 迅速作出反应，所以说，将人工智能应用在计算机网络技术中，可以有效保障信息安全，有助于计算机网络安全管理。

（2）计算机本身对外来信息的安全性无法进行准确、有效的判断，而通过对人工智能的应用，则能够大大提 高计算机对外来信息安全性的判断能力, 使计算机对每一个外来信息都能够作出准确有效的安全性分析，这样能够 使计算机信息系统得到更加有效的保护，同时，还能够有效增强计算机本身的防护墙、入侵检测以及反垃圾邮件能 力。通过对人工智能系统的应用，能够使计算机防护墙变得更加智能化，从而起到更好防护效果。智能系统具有 较强的识别功能, 可以实现对外来病毒以及干扰信息的迅速、准确识别, 且会做出迅速反应, 从而有效防止病毒 以及各种干扰信息的入侵。此外，智能信息系统还可以对邮件进行甄别与筛选，能够及时发现并篮选出垃圾邮件， 这样不仅不会影响用户的正常使用，还会对计算机系统起到很好的防护作用。

\section{2 人工智能在计算机网络系统管理及评价中的应用}

要想使计算机网络技术与人工智能更加有效的结合, 必须要做好其功能以及服务等方面的对接, 因此, 人工智 能系统更多的是在网络技术中承担一个数据快速采集和深入分析的角色, 人工智能在计算机网络技术中起到积极作 用的同时, 计算机网络技术也会在人工智能中起到重要作用, 只有加强对人工智能与计算机网络技术的结合, 才能 促进两者的发展，才能使两者的作用得到更加充分的发挥。

结语

综上所述，我们不难发现人工智能与计算机网络技术之间的结合是必然的，随着科学技术的快速发展，人工智 能与计算机网络技术也必然会得到更加充分的结合。因此, 研究人员应当加强对智能系统的研究, 对智能系统进行 不断的完善，从而使人工智能在计算机网络技术中发挥出更加重要的作用。

[参考文献]

[1]朱俊波.探讨人工智能在计算机网络技术中的应用 [J].科技传播, 2017,9 (04) : 21-22.

[2] 马越. 探讨人工智能在计算机网络技术中的应用 [J]. 计算机光盘软件与应用, 2014, 17 (22): 43-44.

[3] 张涁.探讨人工智能在计算机网络技术中的应用 [J].软件, 2012,33(11): 265-266.

[4] 熊英. 人工智能及其在计算机网络技术中的应用 [J]. 技术与市场. 2011 (02): 28.

[5] 陈斌. 人工智能在计算机网络技术中的应用 [J].技术与市场. 2010 (12): 66 .

作者简介: 孙本楠, 男, 85 年生, 北京, 硕士学历, 工作于北京东方计量测试研究所, 工程师。 